\title{
Industrial dimensions of food allergy
}

\author{
René Crevel \\ SEAC Toxicology Laboratory, Unilever Research, Sharnbrook, Bedford MK44 1LQ, UK
}

\begin{abstract}
Serious attempts to estimate the impact of allergic reactions to foods on public health did not begin until the 1980s. Until about 15 years ago food allergy was considered a minor aspect of food safety. Two developments probably prompted a radical re-appraisal of that situation. The first was the apparently inexorable rise in the prevalence of atopic diseases, of which food allergy forms a part, with its possible consequences highlighted by some well-publicised severe reactions. The second was the growth of genetic modification technology, manifested by the commercialisation of transgenic crops. Each of these developments impacted on the food industry in distinct ways. On the one hand, consumers with food allergies had to be enabled to avoid specific allergens in products formulated with existing ingredients. Food manufacturers therefore had to identify those specific allergens down to trace amounts in all the ingredients forming the product and label or remove them. On the other hand, the introduction of products using ingredients from novel sources required an assessment of the allergenicity of these ingredients as an integral part of safety assurance. The approaches used by the food industry to protect existing consumers who have food allergies and those at potential risk of sensitisation from novel proteins will be illustrated, emphasising how they need to be built into every stage of the life cycle of a product.
\end{abstract}

Allergen management: Industry: Legislation

Food allergy has been long recognised as a clinical phenomenon, with numerous reports in the 20th century medical literature (Prausnitz \& Küstner, 1921; Tuft \& Blumstein, 1942; Loveless, 1950). However, while it was known that patients could suffer extremely severe and sometimes fatal reactions following ingestion of minute amounts of the offending food, food allergy was perceived as a problem for the individual sufferers. Over the last two decades, however, this perception has changed and food allergy is now recognised as a public health problem. A major factor in this increased concern is probably the rise in the prevalence of atopic disease (Lewis et al. 1996), of which it can be considered a manifestation. The prevalence and incidence of food allergy and the number of severe reactions (Sheikh \& Alves, 2000) also appear to be increasing, although the lack of sound baseline epidemiological data still precludes firm conclusions. The increasing interest has driven action by food manufacturers, retailers and regulatory bodies to ensure that the risk to individuals who have food allergies is minimised. This action has been reflected in new legislation in Australia and New Zealand (Food Standards Australia New Zealand, 2002), the EU (European Commission, 2003) and the USA (US Congress,
2004). It has also helped to shape the regulatory requirements for the introduction of new proteins to the market; particularly, but not exclusively, those proteins produced by modern biotechnology. The present paper reviews the ways in which food allergy has affected the food industry and how it has met the challenges posed.

\section{Dimensions of food allergy}

The term 'dimension' refers to two different concepts: size, i.e. how big is the problem, how many individuals does it affect; scope, i.e. what are the limits and nature of the problem. Both concepts are relevant to the current discussion

The size of the food allergy problem relates to the prevalence of the condition and to the extent to which it affects quality of life. Until fairly recently a consensus existed of a prevalence of 1-2\% of the overall population, increasing to 5-8\% of children (Bock \& Atkins, 1990; Young et al. 1994) in whom food allergy is in part a phase of the development of an allergic condition (Rhodes et al. 2002). In the EU this level of prevalence represents about 5-10 million in the population. However, recent data from 
France (Kanny et al. 2001) and the USA (Sampson, 2004) suggest prevalence rates of 3-4\% of the total population. Furthermore, the number actually affected will be severalfold larger, including, for example, members of the immediate family or household. Prevalence of selfreported food allergy is usually many times greater than true prevalence estimated from clinical measures (Young et al. 1994). The potential direct impact on the food industry through loss of sales, for example, is therefore greater than suggested by true prevalence values, although it would be difficult to quantify. A different way of looking at the size of the food allergy problem is to consider the resources deployed to address it or the impact of compliance activities by different authorities. Thus, the US Food and Drug Administration recorded >100 allergen-related recalls per year in the period 1996-2000. A large-scale recall, such as occurred in the context of Starlink $^{\mathrm{TM}}$ maize (a GM variety not approved for human consumption that was found in many parts of the US food supply), can cost a company several hundred million dollars (see Lin et al. 2001).

The scope of food allergy encompasses many elements, including the manifestations and consequences of the disease, assessment of the risk and how to manage it. The extent to which these elements impact on different stakeholders varies but, ultimately, the management of allergen risks is a shared responsibility of all the stakeholders. Health professionals diagnose and advise patients, who in turn must exercise due care in their food and product choices, while food authorities must balance the interests of all stakeholders and ensure compliance.

The food industry's responsibility is to provide safe foods for all intended consumers. Preventing sensitisation to existing common allergens would imply the removal of many wholesome foods from the food supply and is not generally an option. However, food manufacturers can help individuals who know that they suffer from a food allergy by informing them when the specific allergen is present in a product (labelling), or by ensuring that it is not present at a level that will cause harm to those consumers. While labelling or removing specific allergens from foods fully discharges the food manufacturers' responsibility in relation to commonly-allergenic foods, society demands a more stringent test where novel proteins or foods are concerned. Specifically, the novel protein or food should not provoke reactions in individuals who are allergic to a specific allergen, and should also be shown to have little or no potential to produce IgE-mediated immune responses.

\section{Challenges for the food industry}

Several obstacles lie in the way of achieving these objectives. The most important objective, in relation to existing allergens, is that total absence of a specific allergen in foods where it is not intended to be present is often very difficult to achieve because of manufacturing practices. For instance, few production lines are used to manufacture a single product. To produce a safe product the manufacturer therefore has to ensure that any residual allergen is below the level at which it might provoke reactions, even in highly-sensitive individuals. However, individuals who are allergic to a food protein vary greatly in their sensitivity, with very low amounts documented as causing reactions (Foucard \& Malmheden Yman 1999; Taylor et al. 2002). The lowest eliciting dose (threshold) is therefore very difficult to define, although it is at least accepted that such thresholds exist (Taylor et al. 2002). This situation affects the decision to label, which must be driven by the need to protect consumers rather than by the continuous refinement of analytical techniques and, hence, the ability to detect ever more minute traces of allergen. It also influences the measures that must be taken in order to reduce or avoid cross-contact, and the stringency, and therefore the cost, will depend, other factors being equal, on the lowest level of allergen that can be tolerated. Such measures can reach a point of diminishing returns at which additional resources bring little additional protection. An interesting and unresolved issue about thresholds is whether repeated stimulation of some exceptionally-sensitive individuals, through ingestion of residual allergens in sub-threshold amounts, could result in such individuals becoming ultimately primed to react to those levels. Although the physiology of IgE-mediated reactions gives biological plausibility to such a scenario (Arps et al. 1998; Rogers \& Croft, 1999), there is currently no clinical evidence to support this hypothesis. However, should evidence emerge to support it, current risk-assessment paradigms for food allergens would need to be revised. Another issue that is unclear at present is the relationship between the sensitivity and the severity of reaction. It is usually implicit in discussions that the mostsensitive individuals are also those who experience the most severe reactions, but this association is by no means formally proven.

The phenomenon of cross-reactivity between allergens, sometimes from unrelated sources, also challenges the aim to make foods safe for individuals who have food allergies. While many instances have been demonstrated experimentally (e.g. fruits cross-reacting with latex, such as avocado and banana; Wagner \& Breiteneder, 2002), their clinical significance has not been studied to the same extent, and data are therefore lacking to assess potential risks from this type of exposure.

Another obstacle relates to demonstrating that a novel protein is not a potential allergen. Currently, no single predictive test exists to identify and rank protein allergens, and the knowledge about the relationship between protein structure and allergenicity is not adequate for the prediction of protein allergenicity (Aalberse, 2000). Knowledge of the functional family to which a protein belongs can help form a judgement on the probability of it being allergenic, but is not sufficiently predictive on its own (Breiteneder \& Mills, 2005). The current paradigm is, therefore, that any novel protein is considered a potential allergen and must be assessed.

\section{Food industry approach to allergen risk management}

\section{Management of known allergens}

Current approaches to the management of the allergen risk in the food industry recognise that it must be integrated 
into the whole product life cycle from design right through to the point at which the consumer eats it. Major food manufacturers have devised specific corporate policies for the handling of allergens, supplemented by guidelines that provide practical advice to individual manufacturing units. These methods ensure that a high minimum standard exists for the handling of allergens throughout the company. For instance, Unilever has a policy for dealing with allergens that states that it shall declare the presence in its products of any allergen that is a common cause of allergic reactions. At a minimum, any allergen required by local regulations will be declared. However, beyond that undertaking, the allergenic risk from foods not commonly known to be allergenic may be assessed if clinical or epidemiological data indicate the need. If then classed as a common cause of allergic reactions, this food component would then be declared on labels and included in hazard analysis and critical control point (Food and Agriculture Organization, 1998) plans. Unilever also undertakes to inform any consumer who asks about the presence of uncommon allergens in specific products.

As previously mentioned, there are basically two ways of protecting consumers who have food allergies: the first is to inform them; the second is to ensure that the allergen is not present in products at levels sufficient to cause harm. Information is provided by appropriate labelling and should therefore be readily understood by the consumer, which means using generally understood terms, not technical ones, to name allergenic components. For instance, the term 'milk proteins' is preferable to 'whey powder' or 'casein', even though the latter are more technically accurate.

The second component involved in protecting the consumer who has food allergies is to avoid the inadvertent presence of allergen in a product. Like most food manufacturers, Unilever has prepared guidelines to help avoid the inadvertent presence of specific allergens in food products. These guidelines illustrate well how this process requires consideration of all stages in the product life cycle, from its design, through the sourcing of ingredients to manufacture, labelling and distribution. Specifically, it needs to deal with:

innovation: is the use of the allergenic ingredient necessary for the functionality of the product, or could an equivalent non-allergenic ingredient serve as well? supply chain: control of allergens in the supply chain requires a close relationship with suppliers, so that they understand the manufacturer's needs and can meet their requirements. Typically, the basis of the supplier assessment will be a questionnaire about allergens handled and precautions in place to avoid cross-contact, including the existence of a hazard analysis and critical control point plan. This questionnaire is backed up by periodic audits of the suppliers' facilities. Additionally, suppliers are required to seek agreement to any change in the formulation of the ingredient they supply.

manufacturing protocols: these protocols are another critical element. The main considerations are: the inclusion of common allergens in hazard analysis and critical control point plans; production scheduling to minimise cross-contact; validated cleaning procedures; clear labelling and separation of specific allergenic ingredients within the factory. Procedures need to cover 'rework', i.e. where sound product is not packaged but 'recycled'. Training staff to understand the importance of allergen control procedures is vital and improves support for what can be additional procedures in the production process. Finally, the same extent of attention is needed whether the company's own manufacturing facility is concerned or whether third-party manufacturers are used.

packaging, promotion and advertising: packaging carries the label and therefore the allergen information. Care is required to ensure that information remains with the product until it reaches the consumer. Other considerations include warnings if the formulation has changed to include an allergenic ingredient previously not present.

retailers: generally, the manufacturer's allergen information will be sufficient. However, situations such as in-store promotions require care to ensure that the consumer is fully informed. Sound product that fails to meet all standards for general sale may be repackaged and sold on in specialised outlets or even in a different market. The manufacturer needs to ensure that appropriate allergen information is retained and available to the ultimate consumer.

food professionals: most allergic reactions to foods occur outside the home, in conditions where the product is often not labelled, and even when asked food professionals fail to provide correct information. Where pre-prepared food is provided to that sector, the manufacturer has a responsibility to ensure that accurate allergen information is provided and conveyed to the consumer.

\section{Minimum eliciting doses (thresholds) in food-allergen risk management}

Understanding how much of an allergenic food is required to provoke a reaction is a key element in being able to assess the risk and design appropriate allergen management measures. Formal determination of the lowest eliciting dose for a population with food allergy starts with controlled clinical studies, using a double-blind placebocontrolled food challenge protocol. In classical toxicological terms such studies have the advantage of being performed in the species of interest, i.e. human subjects. However, the logistics of these studies mean that subject numbers are, and will probably always remain, fairly small. They may not consequently provide the extent of assurance required for risk assessors. Bearing in mind these considerations, new approaches have been proposed that make fuller use of the information derived from challenge studies (Bindslev-Jensen et al. 2002). These approaches use statistical modelling of the dose distribution of eliciting doses within the study to predict the proportion of the population with food allergy likely to react to specified amounts of allergenic protein beyond 
the lowest experimentally-determined eliciting dose. These statistical models can help quantify the impact of specific allergen risk management measures, and can also aid the discussion of allergen management objectives with other stakeholders. Recently, the application of such models has been critically reviewed (RWR Crevel, D Briggs, SL Hefle, AC Knulst and SL Taylor, unpublished results), highlighting the need for validation before they are applied. Salient questions that this validation process needs to address include whether the subjects participating in double-blind placebo-controlled food challenge trials are representative of the whole clinical population, to what extent are allergic reactions unreported and whether individuals who have food allergies eat broadly the same type of product at the same frequency as individuals who do not have food allergies, which has implications for assessing exposure. Another important piece of data will be the level of undeclared allergen in foods currently consumed safely by individuals with food allergies (e.g. cross-contact allergen).

\section{Assessment of the potential allergenicity of novel proteins and foods}

The introduction of novel proteins requires an assessment of their potential allergenicity. Current strategies consist of systematically building up a body of evidence in order to form a judgement on the allergenicity of the protein (or food) of interest. One of the first such schemes was that proposed by the International Life Sciences Institute International Food Biotechnology Council (Metcalfe et al. 1996), which has been revised and updated by a consultation organised by Food and Agriculture Organization/ World Health Organization (2001). The latter recommendations have since been revised by the Codex Alimentarius Commission (2002). All these schemes contain common elements, which include sequence analysis, investigation of IgE binding and resistance to proteolysis in the presence of gastric enzymes. Analysis of the primary sequence of the proteins serves to identify global similarity with known allergenic proteins or the presence of short matching sequences that would be possible epitopes. Investigation of IgE binding can look at reactivity with other proteins from the same source, or at reactivity with potentially cross-reactive proteins, based on structural or functional similarities. Resistance to pepsin digestion is based on the observation that several well-known allergens hydrolyse relatively slowly in the stomach and hence must reach the immune tissues of the gastrointestinal tract largely intact. Furthermore, all the schemes allow for the possibility of human testing (e.g. skin-prick testing) to confirm lack of reactivity. The schemes differ mainly in how they allow for interpretation of findings. The two earlier schemes (Metcalfe et al. 1996; Food and Agriculture Organization/ World Health Organization, 2001) were proposed as decision trees, which ineluctably led to a specific conclusion, but the current Codex Alimentarius Commission (2002) proposals specifically endorse a weight of evidence approach.

\section{Conclusions}

Food allergy is recognised as an important public health problem. It can have severe and sometimes fatal consequences for a minority of sufferers and impairs the quality of life of other sufferers and their families. In addressing the issue the food industry has faced many challenges. Meeting these challenges has required a systematic consideration of all the elements that contribute to a product from the initial concept to the point at which it reaches the consumer. In particular, much closer links have been developed with suppliers and manufacturing processes have been re-thought to reduce the risk arising from allergens. Nevertheless, gaps remain that place limits on the assessment of allergenic risks, including a lack of information about thresholds of reactivity and of good predictive tools to assess the allergenicity of novel proteins.

\section{References}

Aalberse RC (2000) Structural biology of allergens. Journal of Allergy and Clinical Immunology 106, 228-238.

Arps V, Sudowe S \& Kolsch E (1998) Antigen dose-dependent differences in IgE antibody production are not due to polarization towards Th1 and Th2 cell subsets. European Journal of Immunology 28, 681-686.

Bindslev-Jensen C, Briggs D \& Osterballe M (2002) Can we determine a threshold level for allergenic foods by statistical analysis of published data in the literature? Allergy 57, 741-746.

Bock SA \& Atkins FM (1990) Patterns of food hypersensitivity during sixteen years of double-blind, placebo-controlled food challenges. Journal of Pediatrics 117, 561-567.

Breiteneder H \& Mills ENC (2005) Molecular properties of food allergens. Journal of Allergy and Clinical Immunology 115, $14-23$.

Codex Alimentarius Commission (2002) Report of the third session of the Codex ad hoc Intergovernmental Task Force on foods Derived from Biotechnology (ALINORM 03/34). ftp://ftp.fao.org/codex/alinorm03/A103_34e.pdf

European Commission (2003) Directive 2003/89/EC of the European Parliament and of the Council of 10 November 2003 amending Directive 2000/13/EC as regards indication of the ingredients present in foodstuffs. Official Journal of the European Communities L308, 15-18.

Food and Agriculture Organization (1998) Food Quality and Safety Systems - A Training Manual on Food Hygiene and the Hazard Analysis and Critical Control Point (HACCP) System. Rome: FAO.

Food and Agriculture Organization/World Health Organization (2001) FAO/WHO Expert Consultation on Foods Derived from Biotechnology. Rome: FAO.

Food Standards Australia New Zealand (2002) The Australia New Zealand Food Standards Code. http://www.foodstandards.gov.au/foodstandardscode/

Foucard T \& Malmheden Yman I (1999) A study on severe food reactions in Sweden - Is soy protein an underestimated cause of food anaphylaxis? Allergy 54, 261-265.

Kanny G, Moneret-Vautrin DA, Flabbee J, Beaudouin E, Morisset M \& Thevenin F (2001) Population study of food allergy in France. Journal of Allergy and Clinical Immunology 108, 133-140.

Lewis S, Butland B, Strachan D, Bynner J, Richards D, Butler N \& Britton J (1996) Study of the aetiology of wheezing illness 
at age 16 in two national British birth cohorts. Thorax 51, 670-676.

Lin W, Price GK \& Allen E (2001) StarLink: Impacts on US corn market and world trade. Feed Yearbook, pp. 46-54. Washington, DC: Economic Research Service, USDA; available at http://www.ers.usda.gov/Briefing/Corn/pdfs/ StarLinkFDS2001.pdf

Loveless MH (1950) Milk allergy: a survey of its incidence; experiments with a masked ingestion test. Journal of Allergy 21, 489.

Metcalfe DD, Astwood JD, Townsend R \& Sampson HA (1996) Assessment of the allergenic potential of foods derived from genetically engineered crop plants. Critical Reviews in Food Science and Nutrition 36, Suppl., S165-S186.

Prausnitz C \& Küstner H (1921) Studien über die Überempfíndlichkeit (Study of hypersensitivity). Zentralblatt für Bakteriologie Mikrobiologie und Hygiene Abteilung Originale 86, 160-169.

Rhodes HL, Thomas P, Sporik R, Holgate ST \& Cogswell JJ (2002) A birth cohort study of subjects at risk of atopy: twentytwo-year follow-up of wheeze and atopic status. American Journal of Respiratory and Critical Care Medicine 165, 176-180.
Rogers PR \& Croft M (1999) Peptide dose, affinity, and time of differentiation can contribute to the Th1/Th2 cytokine balance. Journal of Immunology 163, 1205-1213.

Sampson HA (2004) Update on food allergy. Journal of Allergy and Clinical Immunology 113, 805-819.

Sheikh A \& Alves B (2000) Hospital admissions for acute anaphylaxis: time trend study. British Medical Journal 320, 1441.

Taylor SL, Hefle SL, Bindslev-Jensen C, Bock SA, Burks AW, Christie L et al. (2002) Factors affecting the determination of threshold doses for allergenic foods: How much is too much? Journal of Allergy and Clinical Immunology 109, 24-30.

Tuft L \& Blumstein GI (1942) Studies in food allergy. II. Sensitization to fresh fruits: clinical and experimental observations. Journal of Allergy 13, 574-581.

US Congress (2004) Food Allergen Labelling and Consumer Protection Act of 2004. http://www.cfsan.fda.gov/ dms/ alrgact.html

Wagner S \& Breiteneder H (2002) The latex-fruit syndrome. Biochemical Society Transactions 30, 935-940.

Young E, Stoneham MD, Petruckevitch A, Barton J \& Rona R (1994) A population study of food intolerance. Lancet $\mathbf{i}$, $1127-1130$. 